\title{
Characterization and investigation of NLO properties of some selected electrodeposited polythiophenes
}

\section{Figà \\ viviana_tg@yahoo.it \\ J. Luc \\ B. Kulyk \\ M. Baitoul \\ B. Sahraoui}

\author{
Department of Chemical, Process and Materials Engineering, University of Palermo, Viale delle \\ Scienze, 90128 Palermo, Italy \\ Laboratory POMA, UMR CNRS 6136, University of Angers, 2 Bd Lavoisier, 49045 Angers, France
}

Scientific-Technical and Educational Center of Low Temperature Studies, Ivan Franko National University of Lviv, Dragomanova Street, 50, UA-79005 Lviv, Ukraine

Faculty of Sciences Dhar el Mahraz, B.P. 1796, Fés 30000 - Morocco

In this work we study the electronic properties of $\mathrm{ClO}_{4}^{-}$doped polythiophenes and discuss the nonlinear optical properties of these organic compounds galvanostatically electrodeposited on ITO glasses. The investigation on the electronic properties (band gap, flat band potential) was performed by means of a non-destructive optical technique, photocurrent spectroscopy (PCS). The investigation on the nonlinear optical response was carried out by means of second and third harmonic generation (SHG and THC) measurements. In particular, the effect of the oxidation state of the polymeric films was studied by comparing the NLO response of oxidized and reduced polythiophenes. Reduced polymeric films show higher values of the second $\left(\chi^{\langle 2\rangle}\right)$ and third $\left(\chi^{\langle 3\rangle}\right)$ order nonlinear optical susceptibilities. [D0I: 10.2971/jeos.2009.09016]

Keywords: band gap, flat band potential, SHG, THG, NLO properties

\section{INTRODUCTION}

Conjugated polymers have been considered interesting materials for applications in different fields such as electronics, photonics, corrosion protection, etc. They present numerous advantages: light-weight, high chemical inertness, high corrosion resistance, high performance/ low cost, possibility of transitions from metallic to insulating or semiconducting states and vice versa. In particular, semiconducting polymers can be used in the fabrication of field effect transistors, solar cells, lasers, etc. Polymeric materials with large values of second and third order optical susceptibilities are potentially applicable to optical data storage, optical computing, imaging, dynamic holography applications. In addiction, their potential applications are extended also in the field of telecommunications. In fact, polymeric electro-optic materials can function as tunable Bragg wavelength filters, ultra-high bandwidth signal modulators for telecommunications, electricalto-optical signals transducers, controllers of the phase of radiofrequency, etc [1]-[5].

Experiments carried out on polythiophenes (PT) [6] and on several of its derivatives have shown that PTs present a large and very fast NLO response $[7,8]$. In this work, we study the influence of the oxidation state on the NLO properties of some selected polythiophenes galvanostatically electrodeposited on ITO glasses and we characterize the energetics of these compounds.

\section{METHODOLOGY}

Polythiophene thin films were prepared electrochemically, by anodic oxidation of the monomer. The electropolymerization of thiophene was performed using a three electrodes cell connected to a multichannel potentiostat Princeton Applied Research VMP2. The working electrodes were a gold $\operatorname{rod}(S=$ $\left.0.125 \mathrm{~cm}^{2}\right)$ for the PCS studies and indium doped-tin oxide (ITO) glasses $\left(R \leq 7 \Omega \mathrm{cm}^{-2}\right.$; thickness $=1.1 \mathrm{~mm}$ ) by Praezisions Glas \& Optik $\mathrm{GmbH}$ for the nonlinear optical response investigation. The counter electrode was platinum and the reference electrode was a saturated calomel electrode (SCE). Thiophene (Aldrich, 99.5\%) was dissolved in acetonitrile (Aldrich, 99\%) HPL grade anhydrous and $0.1 \mathrm{M}$ lithium perchlorate (Aldrich, 99.9\%) was used as supporting electrolyte. The monomer concentration was $0.1 \mathrm{~mol} / \mathrm{L}$. Before the electropolymerization, the gold electrode and the ITO glasses were sonicated in acetone for 10 minutes. Electropolymerization was performed galvanostatically, applying a constant current density of $0.8 \mathrm{mAcm}^{-2}$. The samples used for the PCS test were prepared with an electrodeposition charge of $95 \mathrm{mCcm}^{-2}$.

In order to investigate on the possible dependence of the NLO properties with the oxidation state, two kinds of samples were prepared: oxidised and reduced polythiophenes. All the samples differ for the circulated charge during the electrodeposition (from 95 to $380 \mathrm{mCcm}^{-2}$ ). For the reduced samples, the 


\begin{tabular}{|c|c|c|c|}
\hline Compounds & $\begin{array}{c}Q_{\text {electropol }} \\
\text { [mCcm }^{-2} \text { ] }\end{array}$ & $\begin{array}{c}Q_{\text {red }} \\
{\left[\mathbf{m C c m}^{-2} \text { ] }\right.}\end{array}$ & $\begin{array}{c}\text { Thickness } \\
{[\mathbf{n m}]}\end{array}$ \\
\hline A & 95 & - & 190 \\
\hline B & 190 & - & 580 \\
\hline C & 284 & - & 760 \\
\hline D & 380 & - & 1160 \\
\hline E & 95 & 95 & 210 \\
\hline F & 190 & 95 & 640 \\
\hline G & 284 & 95 & 650 \\
\hline H & 380 & 95 & 820 \\
\hline
\end{tabular}

TABLE 1 Summary of the different polythiophene films doped with $\mathrm{ClO}_{4}^{-}$in acetonitrile solutions.

reduction charge was $95 \mathrm{mCcm}^{-2}$ (see Table 1) and the applied current density was $0.5 \mathrm{mAcm}^{-2}$. The measurements of the experimental thickness were carried out using a surface profiler VEECO Dektak 6M.

For the characterization of the energetics of polythiophene films, photocurrent spectroscopy (PCS) studies were carried out. In Figure 1, the PCS set up is shown.

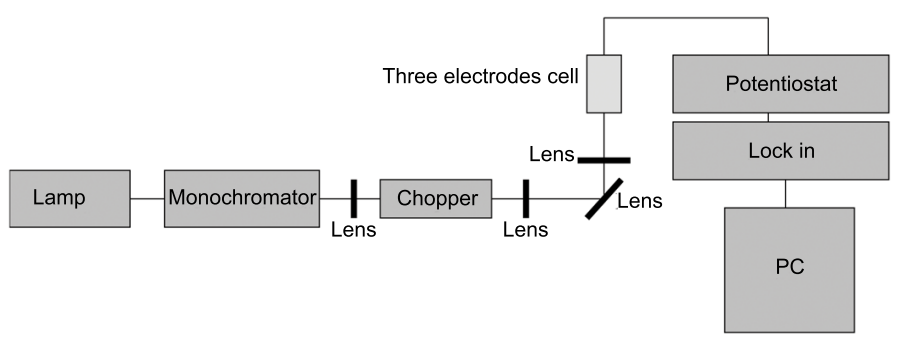

FIG. 1 Experimental set up for PCS studies.

Light is generated by a $450 \mathrm{~W}$ UV-vis xenon lamp (Xenonlampenversorgung Typ SVX 1450, Müller) coupled with a monochromator (Kratos) that allows to select a specific wavelength $(\lambda)$ or to vary it with different scan rates. A series of lenses is used to focus the light beam on the sample. As photocurrent is the difference between the total current and the current in dark condition, a mechanical chopper (Model 650 Light Chopper Controller, EG\&G Instruments) was used. It separates the current under dark conditions from the total current, with a frequency of $13 \mathrm{~Hz}$. The photocurrent is detected by a two-phases lock in amplifier (Model 7260 DSP, EG\&G Instruments) in connection with the mechanical chopper. A three electrodes cell endowed with quartz windows was used in order to avoid the absorption of high-energy photons $(h v \geq 4 \mathrm{eV})$. An $\mathrm{Ag} / \mathrm{AgCl}$ electrode and a platinum foil were employed as reference and counter electrodes respectively. Both photocurrent spectra $\left(I_{p h}\right.$ vs $\left.\lambda\right)$ and photocharacteristics $\left(I_{p h}\right.$ vs $\left.\mathrm{U}_{E}\right)$ were recorded.

SHG and THG measurements were performed in air by the Maker fringe technique [9] for the $s-s$ polarization state (the $s-p$ and $p-p$ polarization states have given a more weak sensitivity of SHG and THG measurements). In Figure 2, the experimental set up is shown.

A quartz $y$-cut plate $\left(\chi^{\langle 2\rangle}=1.0 \mathrm{pmV}^{-1}\right.$ [10]) has been used as a reference material for SHG and fused silica plate $\left(\chi^{\langle 3\rangle}=\right.$

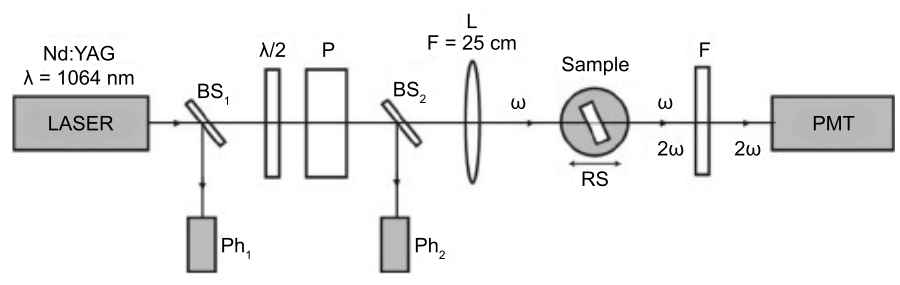

FIC. 2 Experimental set up for SHG measurements: $\left(\mathrm{BS}_{1}, \mathrm{BS}_{2}\right)$ beam splitters, $\left(\mathrm{Ph}_{1}\right.$, $\mathrm{Ph}_{2}$ ) photodiodes, $(\lambda / 2)$ half-wave plate, $(\mathrm{P})$ Clan polarizer, $(\mathrm{L})$ convergent lens, (RS) rotation stage, (F) filter(s), and (PMT) photomultiplier tube.

$0.02 \times 10^{-20} \mathrm{~m}^{2} \mathrm{~V}^{-2}$ [11]) for THG measurements. As a fundamental beam, we used the output beam of a Q-switched $\mathrm{Nd}$ :YAG laser, generating a coherent beam at $\lambda=1064 \mathrm{~nm}$ with 16 ps pulse duration and $10 \mathrm{~Hz}$ repetition rate. A halfwave plate seated before the polarizer controlled the polarization and the power density of the fundamental beam. The intensity at the input surface of the sample is assumed to be spatio-temporal Gaussian distribution. The beam diameter was $0.5 \mathrm{~mm}$ on the film and the applied power density was approximately $5 \mathrm{GWcm}^{-2}$. The beam was focused onto the sample with lens of $250 \mathrm{~mm}$ focal length. A motorized rotation stage with the mounted sample allowed the variation of the incident angle with a resolution of $0.5^{\circ}$. After the sample, an interferential filter was placed to select before the photomultiplier (Hamamatsu R1828-01), the harmonic wave (at $532 \mathrm{~nm}$ for SHG or $355 \mathrm{~nm}$ for THG). Detector saturation was prevented using linear neutral density filters, whose transmittance value was taken into account during data fitting. Finally, we got the SHG and THG signals by rotating the sample through a range of $\pm 80^{\circ}$ to the normal. All the experiments were performed at room temperature.

\section{RESULTS}

\subsection{Photocurrent spectroscopy results}

When a semiconductor is irradiated by photons with suitable energy $\left(h v>E_{g}\right)$, it absorbs energy and there is a promotion of electrons from the valence to the conduction band. This phenomenon is called optical transition or phototransition. There are different kinds of optical transitions according to the electronic structure of semiconductors. In crystalline materials, the light absorption occurs by charge carriers inside the bands (intraband transitions). It is also possible that the light absorption is caused by interband optical transitions; in this case the creation of electron-hole pairs takes place. The optical transition from the valence to the conduction band can be direct or indirect according to the dependence of the energy on the electron quasi-momentum in the conduction band. In particular, the direct optical transitions come from the promotion of an electron from the valence to the conduction band by interaction with a photon. In this case, there is not a variation of the electron quasi-momentum. The indirect optical transitions, instead, are characterized by the variation of the electron quasimomentum. In the amorphous materials it happens by the interaction of an electron with a photon [12].

When a semiconductor is in the condition of flat band, recombination processes of photoexcited electrons and holes oc- 
cur easily. In presence of a space charge region, electrons and holes are separated by an electric field, so they move towards the electrode surface and they can participate to charge transfer reactions: electrons for the cathodic reaction - holes for the anodic reaction [13]. The resulting electric current is called photocurrent.

Photocurrent spectra and photocharacteristics of polythiophene electrodeposited on gold, were recorded in $0.1 \mathrm{~mol} / \mathrm{L}$ $\mathrm{LiClO}_{4}$ in acetonitrile. No photocurrent spectra were recorded at anodic potentials such as $U_{E}=0.5 \mathrm{~V}(\mathrm{Ag} / \mathrm{AgCl})$ and $U_{E}=0.1 \mathrm{~V}(\mathrm{Ag} / \mathrm{AgCl})$. The samples are photoactive just at cathodic potentials as $U_{E}=-0.8 \mathrm{~V}(\mathrm{Ag} / \mathrm{AgCl})$ and $U_{E}=$ $-1.2 \mathrm{~V}(\mathrm{AgAgCl})$. Under cathodic polarization, a depletion layer is formed in the polymeric film. Illumination produces electron-hole pairs which are separated by the electric field across the space charge region. Electrons move towards the polymer/electrolyte interface and reduce a redox couple in solution. Holes move towards the polymer bulk and give rise to a cathodic photocurrent. The photoactivity just at cathodic potentials is typical of p-type semiconductors. The lack of anodic photocurrent suggests a metallic or a strongly degenerate p-type semiconducting behaviour.

In Figure 3 is shown the photocurrent spectrum recorded at $U_{E}=-1.2 \mathrm{~V}(\mathrm{Ag} / \mathrm{AgCl})$ not correct for the efficiency of the system lamp-monochromator. The sample is photoactive in a wavelength range between $240 \mathrm{~nm}$ and $650 \mathrm{~nm}$. The maximum value of photocurrent is around $500 \mathrm{~nm}$.

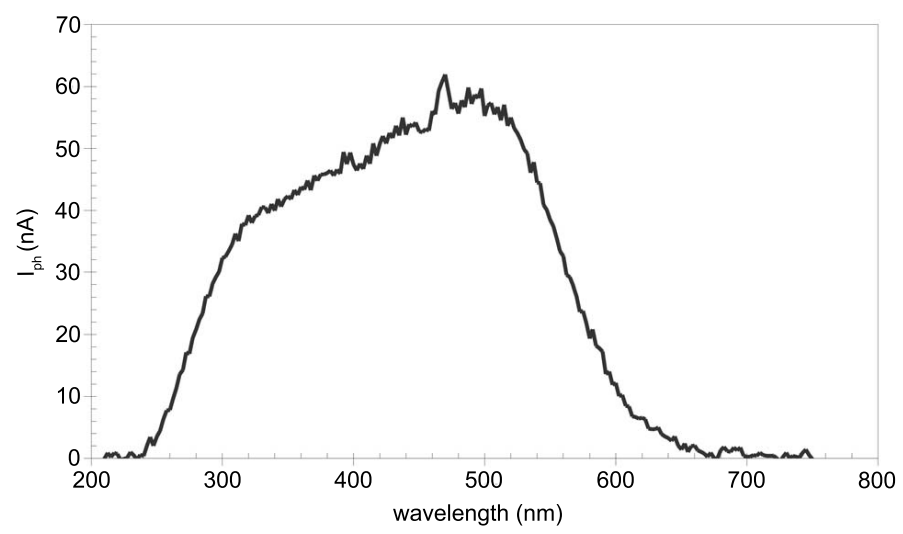

FIG. 3 Uncorrect photocurrent spectrum at $\mathrm{U}_{E}=-1.2 \mathrm{~V}(\mathrm{Ag} / \mathrm{AgCl})$.

For the study of the optical transitions occurring in the polythiophene film, we used the following equation:

$$
\alpha(v)=\frac{A\left(h v-E_{g}\right)^{n}}{h v}
$$

where $\alpha(v)$ is the absorption coefficient, $\mathrm{A}$ is a constant, $h$ is Planck's constant, $v$ is the frequency of the incident light, $E_{g}$ is the optical band gap value of the semiconductor and $n$ is the power law coefficient. $n$ assumes the values 0.5 and 2 for direct and indirect (or non-direct) optical transitions respectively. Eq. (1) represents a law of proportionality between the photocurrent corrected for the efficiency of the system lampmonochromator and the absorption coefficient. In Figure 4, the extrapolations of Eq. (1) for $n=0.5$ and $n=2$ in the case of the corrected spectrum at $U_{E}=-1.2 \mathrm{~V}(\mathrm{Ag} / \mathrm{AgCl})$ are shown for the region of low energies.
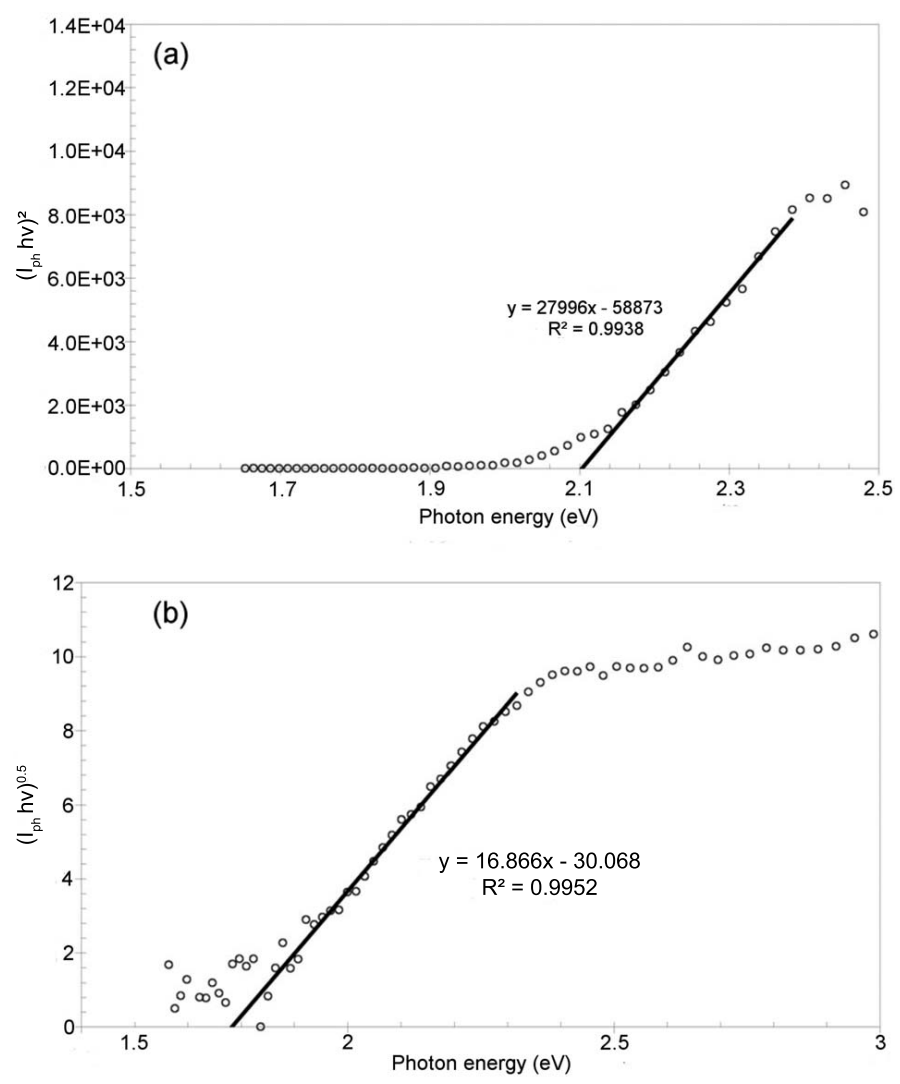

FIG. 4 Extrapolations of Eq. (1) for the corrected photocurrent spectrum at low energies recorded at $\mathrm{U}_{E}=-1.2 \mathrm{~V}(\mathrm{Ag} / \mathrm{AgCl})$ of polythiophene film grown on gold. (a) for $n=0.5$; (b) for $n=2$

Both direct and non-direct or indirect transitions are possible. For $n=0.5$ we obtained the direct band gap of $2.1 \mathrm{eV}$, for $n=2$ the non-direct band gap of $1.78 \mathrm{eV}$. The value of the direct band gap is in agreement with literature [14]. The optical transitions corresponding to $2.1 \mathrm{eV}$ can be attributed to the promotion of the electrons from the bonding $(\pi)$ to the antibonding $\left(\pi^{*}\right)$ orbitals. So it represents the HOMO-LUMO gap of the polymer. The value of the indirect band gap can be attributed to the low-energy optical transitions, which occur from HOMO to a defects band located in the energy gap. This defects band can be polaronic or bipolaronic.

Photocurrent $v s$ applied potential plots (photocharacteristics) were recorded irradiating the samples with light of constant wavelength. The scan rate of potential was $10 \mathrm{mVs}^{-1}$ towards the anodic values.

In Figure 5 are shown the photocharacteristics of polythiophene films recorded at $400 \mathrm{~nm}$ and $500 \mathrm{~nm}$. There are no differences in the shape of $I_{p h}$ against the applied potential (V). The potential at which the value of photocurrent is zero, is called flat band potential $\left(V_{f b}\right)$. From the estimation of $V_{f b}$, it is possible to locate the Fermi level, according to the following:

$$
E_{F}=-|e| V_{f b}
$$

Electrodeposited polythiophene has a flat band potential of $0.42 \mathrm{~V} / \mathrm{NHE}$. 


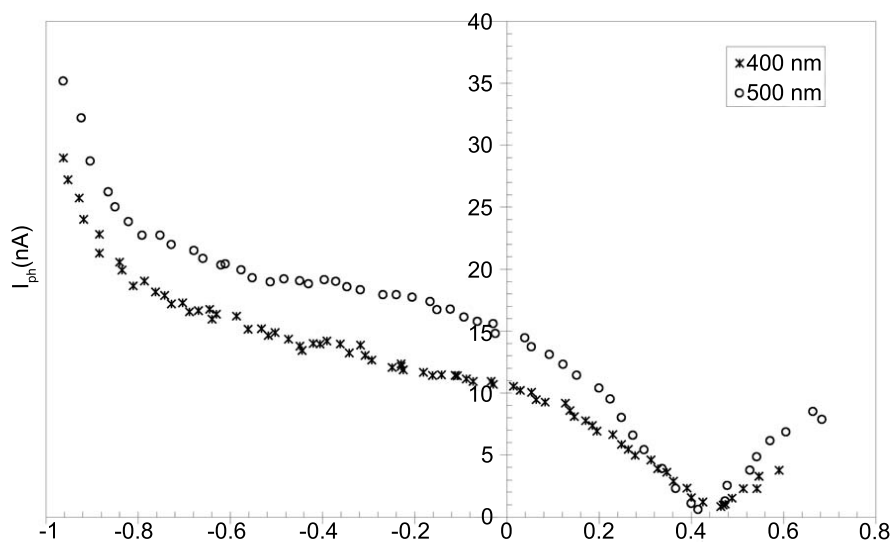

FIG. 5 Photocharacteristics of polythiophene films at $400 \mathrm{~nm}$ and $500 \mathrm{~nm}$.

\subsection{NLO properties of electrodeposited polythiophenes}

Nonlinear optical properties of metallic and semiconducting polythiophenes were studied using second and third harmonic generation measurements. For this reason, oxidised and reduced samples were electrodeposited on ITO glasses according to different experimental conditions. In Table 1, the summary of the different sample preparations is shown.

$Q_{\text {electrodep }}$ is the charge circulated during the electrodeposition and $Q_{\text {red }}$ is the charge circulated during the process of reduction. The samples with different thicknesses correspond to different charges of electrodeposition, according to Faraday's law. In Table 1, the values of the experimental thicknesses are reported. During the process of electrodeposition, oxidation, coupling and deprotonation reactions take place [6]. As the polymer assumes a positive charge, the migration of anions from the solution to the polymer occurs in order to preserve electroneutrality. In the case of conducting polymers, this phenomenon is called doping. On the contrary, by applying a cathodic current density or a cathodic potential, the polymer is reduced and the anions move from the polymeric matrix to the solution. This process is called dedoping and it corresponds to the discharge of the compound.

The SHG measurements were performed by Maker fringe technique for the $s-s$ polarization state. SHG is a nonlinear optical process in which the fundamental beam at a frequency $\omega$ interacts with a nonlinear medium and results in the generation of an additional beam with frequency $2 \omega$. The secondorder susceptibility was calculated by comparing the $\mathrm{SH}$ intensity obtained for the samples and for a reference material (quartz $y$-cut). The average orientation of the compounds was estimated comparing the experimental results with the theoretical angular dependence of the $\mathrm{SH}$ intensity. In Figure 6, the SHG intensity against the incident angle is reported for semiconducting polythiophenes.

All of them show the SHG signal, which is symmetric with respect to $0^{\circ}$ and a maximum intensity between $40^{\circ}$ and $45^{\circ}$ with respect to the incident beam. It means that the chromophoric groups are oriented perpendicular to the surface. Among these compounds, compound F gives a better SHG response than the others but we must take into account the

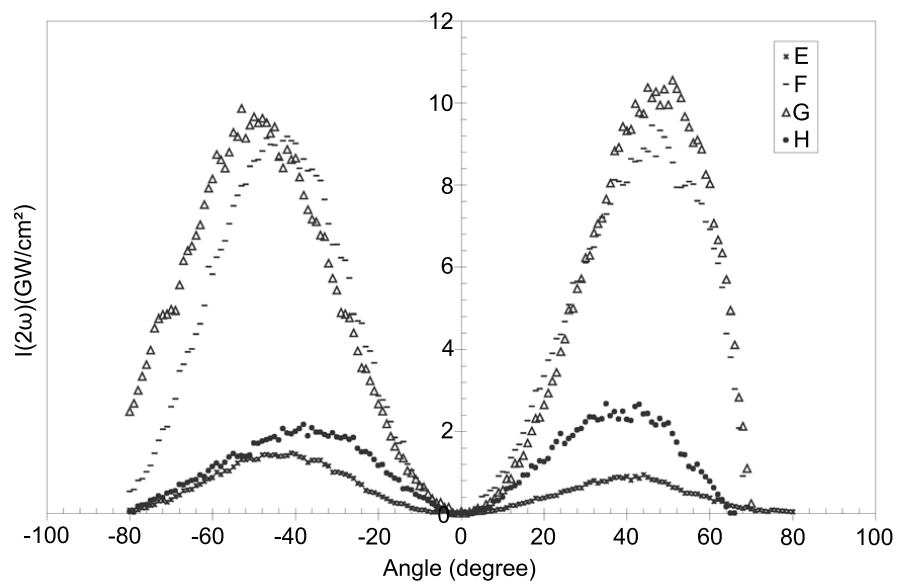

FIG. 6 SHG signal vs incident angle for the reduced samples.

thickness of the compounds to determine the effective nonlinear second-order susceptibility. In general, reduced samples give better SHG signals than the oxidised ones [15]. The values of second order nonlinear susceptibilities $\chi^{\langle 2\rangle}$ were calculated by means of the following equation [16]:

$$
\left|\chi^{\langle 2\rangle}\right|=\left|\chi_{Q}^{\langle 2\rangle}\right|\left(\frac{2}{\pi}\right)\left(\frac{L_{C, Q}}{l}\right)\left(\sqrt{\frac{I^{2 \omega}}{I_{Q}^{2 \omega}}}\right)
$$

with:

$$
L_{C, Q}=\frac{\lambda_{\omega}}{4 \times\left|n_{Q(2 \omega)}-n_{Q(\omega)}\right|}
$$

where $I^{2 \omega}$ is the maximal SH intensity of the sample, $I_{Q}^{2 \omega}$ the maximal SH intensity of quartz $\left(\approx 2.5 \times 10^{5} \mathrm{Wcm}^{-2}\right.$ at $532 \mathrm{~nm}$ and for a thickness of $0.5 \mathrm{~mm}), L_{C, Q}$ is the coherence length of quartz $(\approx 20.5 \mu \mathrm{m}), l$ the thickness of the sample, $\chi_{Q}^{\langle 2\rangle}$ the second order susceptibility of quartz $\left(1.0 \mathrm{pmV}^{-1}\right)[10], n_{Q(2 \omega)}$ the refractive index of second harmonic wave $(1.547$ at $532 \mathrm{~nm})$ and $n_{Q(\omega)}$ the refractive index of fundamental wave (1.534 at $1064 \mathrm{~nm})$ [17].

The values of $\chi\langle y\rangle$ are summarized in Table 2. Higher values of second order nonlinear susceptibility were found in the case of semiconducting polythiophenes even if negligible with respect to $\chi^{\langle\mathrm{y}\rangle}$ the reference material.

THG is a third order nonlinear optical process in which the fundamental beam at the frequency $\omega$ interacts with a nonlin-

\begin{tabular}{|c|c|c|}
\hline Compound & $\chi_{\text {eff }}^{\langle 2\rangle}\left[\mathbf{p m} \mathbf{V}^{-1}\right]$ & $\chi_{\text {elec }}^{\langle 3\rangle}\left[\mathbf{1 0}^{-20} \mathbf{~ m}^{\mathbf{2}} \mathbf{V}^{-2}\right]$ \\
\hline A & 0.16 & 1.44 \\
\hline B & 0.10 & 0.39 \\
\hline C & 0.05 & 0.06 \\
\hline D & 0.02 & 0.01 \\
\hline E & 0.18 & 2.27 \\
\hline F & 0.15 & 0.62 \\
\hline G & 0.15 & 0.47 \\
\hline H & 0.06 & 0.41 \\
\hline Quartz & 1.0 & - \\
\hline Fused silica & - & 0.02 \\
\hline
\end{tabular}

TABLE 2 Second and third order susceptibilities of the investigated samples. 
ear medium and another beam of frequency $3 \omega$ is generated. The third order susceptibility is the sum of different contributions coming from different physical processes. It can be written as:

$$
\chi^{\langle 3\rangle} \approx \chi_{\text {elec }}^{\langle 3\rangle}+\chi_{\text {mol }}^{\langle 3\rangle}+\chi_{\text {therm }}^{\langle 3\rangle}+\chi_{\text {stric }}^{\langle 3\rangle}
$$

where $\chi_{\text {elec }}^{\langle 3\rangle}$ is the electronic component, $\chi_{\text {mol }}^{\langle 3\rangle}$ the molecular (vibrational and rotational) component, $\chi_{\text {therm }}^{\langle 3\rangle}$ the thermal contribution, and $\chi_{\text {strict }}^{\langle 3\rangle}$ the electrostriction component. These contributions have different response times. For the THG measurements in picosecond regime, just the electronic contribution can be estimated. In Figure 7 are represented the angle dependence of the THG signals for the oxidised sample A. The maximum value of the THG signal is obtained around $0^{\circ}$.

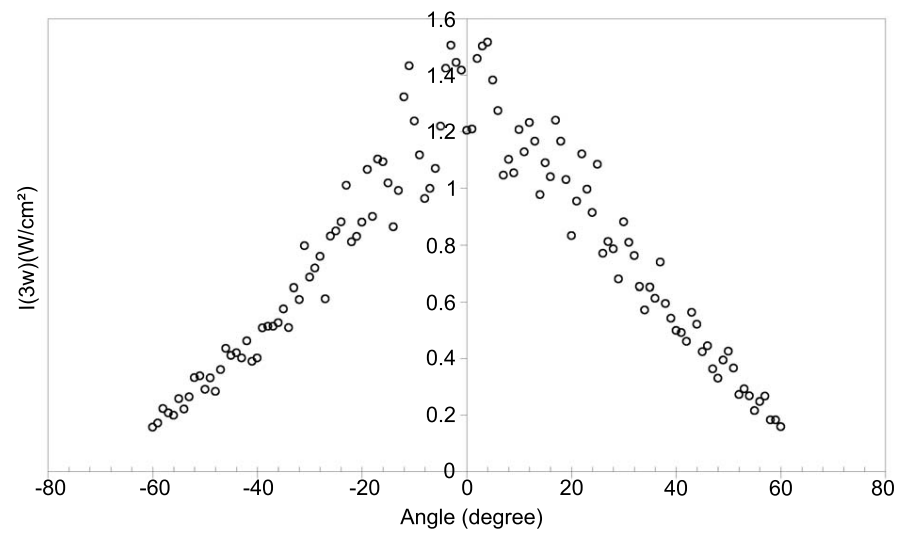

FIG. 7 THG signal vs incident angle for the reduced polythiophenes.

Table 2 summarizes also the values of $\chi_{\text {elec }}^{\langle 3\rangle}$ of both metallic and semiconducting polymers, estimated using the model of Kubodera and Kobayashi [18]. Also for the third harmonic generation response, the higher values were recorded in the case of semiconducting polythiophenes. In particular, compound $E$ shows a value of $\chi_{\text {elec }}^{\langle 3\rangle}$ which is two order of magnitude higher than the $\chi_{\text {elec }}^{\langle 3\rangle}$ value of the reference material.

SHG and THG measurements have been performed in air and the optical absorption at the given wavelength is not taken into account in the formula of the used models. Consequently, the influence of these parameters have induced a measurement uncertainty about $\pm 10 \%$ on the results of second and third nonlinear optical susceptibilities given in Table 2.

Both second and third order nonlinear optical susceptibilities decrease with the increasing of the samples thickness as we expected.

\section{CONCLUSIONS}

In this work, we characterized electrodeposited polythiophenes by means of photocurrent spectroscopy (PCS) and we estimated the values of the second and third nonlinear optical susceptibilities at different oxidation states. From PCS studies it was found that $\mathrm{ClO}_{4}^{-}$doped polythiophenes behave like metals because of the lack of anodic photocurrent and they behave like p-type semiconductors because they are photoactive under cathodic polarization. From the photocurrent spectra, it was found that both direct and non-direct optical transitions are possible for these films. The higher energy optical transitions gave a direct band gap of $2.1 \mathrm{eV}$ while the lower energy ones, an indirect band gap of $1.78 \mathrm{eV}$. From photocharacteristics, it was found that the polymer is under flat band conditions at $0.42 \mathrm{~V} / \mathrm{NHE}$.

The $\chi^{\langle 2\rangle}$ and $\chi^{\langle 3\rangle}$ values have been measured using the SHG and THG methods at the fundamental wavelength of $1064 \mathrm{~nm}$ in picosecond regime. The results showed that semiconducting polythiophenes have got higher values of nonlinear susceptibilities than the metallic ones. Among all, compound $E$ showed a $\chi^{\langle 3\rangle}$ value of $2.27 \times 10^{-20} \mathrm{~m}^{2} \mathrm{~V}^{-2}$ which is two order of magnitude higher than that of fused silica. From a qualitative estimation, therefore from the difference between the charge circulated during the electrodeposition and that of reduction, compound $F$ has the lower doping level. It means that it is possible to improve the third order nonlinear optical properties of polythiophenes by increasing the charge of reduction. The achievement of high nonlinear optical characteristics in organic compounds in general [19]-[21] and particularly in such structures could be perspective for nonlinear optical applications. More detailed studies about the dependence of the NLO properties on the doping level will be performed for these compounds and will be a subject of a next research work.

\section{References}

[1] A. Migalska-Zalas, J. Luc, B. Sahraoui, and I. V. Kityk, "Kinetics of third-order nonlinear optical susceptibilities in alkynyl ruthenium complexes" Opt. Mater. 28, 1147-1151 (2006).

[2] J. Luc, J. L. Fillaut, J. Niziol, and B. Sahraoui, "Large third-order nonlinear optical properties of alkynyl ruthenium chromophore thin films using third harmonic generation" Adv. Mater. 9, 28262832 (2007).

[3] J. Luc, A. Migalska-Zalas, S. Tkaczyk, J. Andriès, J-L. Fillaut, A. Meghea, and B. Sahraoui, "Nonlinear optical effects in new alkynyl-ruthenium containing nanocomposites" Adv. Mater. 10, 29-43 (2008).

[4] B. Kulyk, Z. Essaidi, J. Luc, Z. Sofiani, G. Boudebs, B. Sahraoui, V. Kapustianyk, and B. Turko, "Second and third order nonlinear optical properties of microrod ZnO films deposited on sapphire substrates by thermal oxidation of metallic zinc" J. Appl. Phys. 102, 1-6 (2007).

[5] W. Bi, N. Louvain, N. Mercier, J. Luc, I. Rau, F. Kajzar, and B. Sahraoui, "A switchable NLO organic-inorganic compound based on conformationally chiral disulfide molecules and $\mathrm{Bi}^{(I I I)} \mathrm{I}_{5}$ iodobismuthate networks" Adv. Mater. 20, 1013-1017 (2008).

[6] J. Roncali, “Conjugated poly(thiophenes): Synthesis, functionalization, and applications" Chem. Rev. 92, 711-738 (1992).

[7] P. N. Prasad, J. Swiatkiewicz, and J. Pfleger, “Resonant nonlinear optical processes and charge carrier dynamics in photoresponsive polymers" Mol. Cryst. Liq. Cryst. 160, 53-68 (1988).

[8] V. H. Houlding, A. Hahata, J. T. Yardley, and R. L. Elsenbaumer, "Optical third harmonic response of amorphous poly(3-methyl-4'octyl-2,2'-bithiophene-5,5'-diyl) thin films" Chem. Mater. 2, 169172 (1990).

[9] W. N. Herman and L. M. Hayden, “Maker fringes revisited: secondharmonic generation from birefringent or absorbing materials" J. 
Opt. Soc. Am. B 12, 416-427 (1995)

[10] U. Gubler and C. Bosshard, "Optical third-harmonic generation of fused silica in gas atmosphere: Absolute value of the third-order nonlinear optical susceptibility $\chi^{\langle 3\rangle "}$ Phys. Rev. B 61, 10702-10710 (2000).

[11] F. Kajzar, Y. Okada-Shudo, C. Meritt, and Z. Kafafi, "Second- and third-order non-linear optical properties of multilayered structures and composites of $\mathrm{C}_{60}$ with electron donors" Synth-Int. J. Methods 117 , 189-193 (2001).

[12] Yu. V. Pleskov and Yu. Ya. Gurevich, Semiconductor Photoelectrochemistry (Consultants Bureau, New York, 1986).

[13] H. Gerisher, "The impact of semiconductors on the concepts of electrochemistry" Electrochim. Acta 35, 1677-1699 (1990).

[14] A. K. Bakhshi and S. Kaul, "Strategies for molecular designing of novel low band gap electrically conducting polymers" Appl. Biochem. Biotech. 96, 125-133 (2001).

[15] V. Figà, J. Luc, M. Baitoul, and B. Sahraoui, "NLO properties of polythiophenes galvanostatically electrodeposited on ITO glasses" Adv. Mater. 10, 2123-2128 (2008).

[16] G. J. Lee, S. W. Cha, S. J. Leon, J. I. Jin, andJ. S. Yoon, "Second order nonlinear properties of unpoled bent molecules in powder and in vacuum deposited film" J. Korean Phys. Soc. 39, 912-915 (2001).

[17] R. A. Myers, N. Mukherjee, and S. R. J. Brueck, "Large second-order nonlinearity in poled fused silica" Opt. Lett. 16, 1732-1734 (1991).

[18] K. Kubodera and H. Kobayashi, "Determination of third-order nonlinear optical susceptibilities for organic materials by third harmonic generation" Mol. Cryst. Liq. Cryst. 182, 103-113 (1990).

[19] B. Sahraoui, X. Nguyen Phu, M. Sallé, and A. Gorgues, "Electronic and nuclear contributions to the third-order nonlinear optical susceptibilities of new $\mathrm{p}-\mathrm{N}, \mathrm{N}^{\prime}$-dimethylaniline tetrathiafulvalene derivatives" Opt. Lett. 23, 1811-1813 (1998).

[20] B. Derkowska, J. C. Mulatier, I. Fuks, B. Sahraoui, X. Nguyen Phu, and C. Andraud, "Third order optical nonlinearities in new octopolar molecules and their dipolar subunits" J. Opt. Soc. Am. B 18, 610-616 (2001).

[21] B. Sahraoui, G. Rivoire, J. Zaremba, N. Terkia-Derdra, and M. Sallé, "The third-order nonlinear optical properties of new bis dithiafulvenyl substituted tetrathiafulvalene" J. Opt. Soc. Am. B 15, 923-928 (1998). 